\title{
Digital Innovations in Financial Services: Challenges \& Opportunities
}

\author{
Dr. Aanchal Singhal \\ Assistant Professor, Department of Management Studies, JSSATE, Noida \\ +91 9810953428, aanchalsinghal.dms@jssaten.ac.in \\ Dr. Bindu Arora \\ Professor, Department of Management Studies, Gurukul Kangri University, Haridwar. \\ +919412173247, Binduarora17@rediffmail.com
}

\begin{abstract}
There are many large forces sweeping society, from demographic and social changes to shifts in global economic power. But one force especially-namely, technological breakthroughs - has an inconsistent affect on financial services. Here in this paper important technology-driven influencers which will shape competition in financial services industry are going to be discussed.
\end{abstract}

The financial services industry has seen drastic technology-led changes over the past few years. Many executives look to their IT departments to enhance efficiency and facilitate game-changing innovation - while somehow also lowering costs and continuing to support legacy systems.

Meanwhile, companies are encroaching upon established markets, leading with customer friendly solutions developed from the bottom up and unencumbered by legacy systems. Customers have had their expectations set by other industries; they're now demanding better services, seamless experiences regardless of channel, and more value for his or her money. Regulators demand more from the industry too, and have began to adopt new technologies which will revolutionize their ability to gather and analyze information. The pace of change shows no signs of slowing, in reality technology-driven change is so persistent that no financial organization is immune.

Key Words: artificial intelligence, blockchain, cloud computing, competition, cybercrime. 


\section{Introduction}

\section{Digital Innovations in Financial Services:}

A digital transformation is taking place in the financial services industry. This transformation includes emerging market economies, and in many places offers a viable digital alternative to traditional banks. Financial service companies are facing many challenges today. Most of it has to do with the rapid changes in technology. While the vast majority of financial service companies have embraced the technology revolution, there are still many challenges financial service companies have to face. But, digital innovation also led to many opportunities like instant digital payments, blockchain, artificial intelligence, etc.

\section{Objectives}

- To know the various challenges faced in implementing digital innovations in financial services.

- To find out and understand the opportunities in financial services with development of digital innovations.

\section{Digital Innovations in Financial Services: Challenges}

- Cybercrime:

Data breaches involving financial service firms are increasing. Financial service firms are prime targets for cybercrime. Because of the sensitive data they carry, they are more likely to be targeted. The potential sources of cyber threats and the attack footprint are impossible to eliminate, requiring organizations to be proactive in their approach to cyber security. Financial services executives are already depressingly familiar with the impact that cyberthreats have had on their industry. Unfortunately, it is not likely to change for the better in the coming years, due to the following forces:

Use of third-party vendors

$>$ Rapidly evolving, sophisticated and complex technologies

$>$ Cross-border data exchanges

$>$ Increased use of mobile technologies by customers, including the rapid growth of the Internet of Things

Heightened cross-border information security threats

Cyber-security is already important, and it will become even more significant for institutions and their regulators in the future. The challenge will be to balance safety with customer convenience. With the right tools in place, financial institutions have to improve their ability to manage cyber-risk. For example, financial institutions have to deploy data mining tools and other technologies to detect anomalies in security and fraud applications.

- Regulatory Compliance in Finance

The ever-changing regulatory environment poses a constant challenge for financial institutions of all types which increase the burden of compliance. Regulations in the financial service industry continue to increase. Banks are spending a large part of their income on making sure they're compliant. They have to make sure there are systems in place to keep up with ever-changing regulations and industry standards. Privacy is another critical concern. 
Customers must feel their information will be used to benefit them, and not in a way that intrudes upon their private lives. This is a sensitive topic; in fact, if handled poorly, privacy violations could invite a heavy handed regulatory response. Financial institutions will need strong operational controls in place so data is not being misused.

\section{- Organizing Big Data}

Big data is a necessity but also an obstacle for financial service firms. Big data is getting bigger because a lot of data is being created by a number of sources. This new data is both structured and unstructured, and these legacy data systems can't handle the volume of data coming in. The challenge for financial service companies is to sort through all their data and determine what is useful and what isn't.

\section{- Investment Cost and Adoption of Digital Innovation}

Because of the efficiencies of digital-only competition, banks and credit unions will need to consider divesting from non-core operations and leveraging intelligent automation. In addition, organizations will need to reinvent back office processes and replace aging infrastructure. The financial services sector has been slow to adopt digital transformation. Issues with legacy systems, coupled with often large amounts of data and a reluctance to undertake potentially risky change processes, have meant many firms are behind the curve when it comes to technology adoption.

- Response to customer needs

Financial institutions need to shift from physical interactions to digital engagement as per the needs and requirements of their customers. Today, many financial institutions use cloudbased software-as-a-service (SaaS) applications for business processes that might be considered non-core, such as CRM, HR and financial accounting. They also turn to SaaS for 'point solutions' on the fringes of their operations, including security analytics and KYC verification.

\section{- Weak Infrastructure Support}

Financial Institutions have to prepare their architecture and infrastructure to connect to anything, anywhere. To stay cost-competitive, and to have the flexibility that innovation requires, financial institutions will need to update their infrastructure to make it more agile and responsive. They will need an architecture that can bend as requirements change and interact with data and systems that could be anywhere. And at most financial institutions will require a significant reorientation.

- Updating IT Operating model

Financial institutions may need different hardware, software, or storage technology. They may also need to rethink the way they network the components. Financial institutions and their IT organisations must be prepared for a world where change is constant - and where digital comes first. Financial institutions have a unique struggle though. Will they build or buy? Does location matter? How will they make decisions on what to do, as well as what not 
to do? Do they have the proper organisation to fit the new program, now and as it continues to evolve? How will they know if they are getting it right?

- Access to the necessary talent and skills

Financial institutions will need talent with very different skills. This might involve changes across the human capital strategy through revitalised recruitment, learning and development, partnering and cultural initiatives. Typically, this could include efforts such as:

$>$ Recruiting expert talent from other technology organisations and think-tanks, rather than staying focused on recruiting from within the financial services industry.

$>$ Developing robust learning modules to enhance the skills of executives, IT - and non-IT staff.

$>$ Taking more active steps to create and foster a culture of innovative thinking and talent development.

Engaging more extensively and creatively with third-party sources of talent, including the use of "talent exchanges".

As financial institutions look to the future, one of the biggest hurdles will have nothing at all to do with technology. For years, traditional financial institutions have designed their offerings from the inside out: 'this is what we will offer,' rather than 'what do our customers want?' But this model no longer works. And the skills and interests of today's IT team members and third-party talent may not be up to the challenges of tomorrow's technical environment, where partnering with customers will be essential.

\section{Digital Innovations in Financial Services: Opportunities}

- Instant Digital Payments

Technology has changed consumer and business expectations in payments. The availability of an instant payments platform offers banks an enticing opportunity to achieve the transaction speed consumers expect of their banking experience and increase the customer satisfaction. With instant payments, more transactions will be made digitally instead of in cash, which means that payments will become less expensive and more user friendly. Finally, by expanding and combining instant capabilities with solutions in e- and m-commerce banks and credit unions could develop an innovative portfolio of new services.

- Blockchain

Financial Service firms adopting blockchain technology to improve efficiency, costeffectiveness, and security throughout the entire spectrum of financial services. Some financial institutions have already started testing the use of blockchain for inter-bank transfers, with others testing in the space of payments, fraud reduction, know your customer, and loan processing. Many see tremendous benefits to streamlining and automating processes through smart contracts. Blockchains technology are used in bitcoins, payment transactions, banking industry and other sectors. Cryptocurrency is a medium of exchange, such as the US dollar, created and stored electronically in the blockchain, using encryption techniques to control the creation of monetary units and to verify the transfer of funds. Blockchain has provide various benefits in financial services like increased transparency, accurate tracking, 
permanent ledger and cost reduction. Blockchain systems could be far cheaper than existing platforms because they remove an entire layer of overhead dedicated to confirming authenticity. Blockchain leads to faster, cheaper settlements and could save billions of dollars from transaction costs while improving transparency.

\section{- Surpassing Competition}

Competition within the financial services industry is still very strong. As mentioned earlier, consumers want more personalized service. They also want more automated services with easier access to them. Institutions that provide all these services will dominate their share of the market. Today, consumers are les s concerned with brand loyalty and identity. They just want what they want. Institutions that provide those services will keep their customers.

In India, acceptance of various cashless modes payments was seen after demonetization notes. The government itself encouraged everyone towards the cashless technologies like digital wallets, Internet banking, and the mobile-driven point of sale (POS).

- Keeping up with Technology

Business growth is very important for financial firms, but in order to grow, they must spend money updating their technology. Financial service firms must continue to invest in technology such as robotics and other workflow automation tools to increase their efficiency and reduce the costs associated with operational, risk management, and compliance. Firms must also modernize their technology platforms and data storage so they can enable big data solutions such as AI-supported digital customer support assistants. Financial firms must also consider consolidating platforms and provide a more efficient, customer-friendly experience across internet, mobile and physical locations.

- Artificial Intelligence

With customer data becoming a 'product' for many financial institutions, the need for enhanced security and Artificial Intelligence (AI) will become a differentiator from both a compliance and customer trust perspective. This can lead to reduced costs and potential business growth. Industry experts believe that AI will transform nearly every aspect of the financial service industry. Automated wealth management, customer verification, and open banking all provide opportunities for AI solution providers. Powerful advances in deep learning technology are paving the way for AI. In fact, if you have been alerted by your bank of suspicious activity on your account, you have likely already benefited from AI. ATMs are robots. They are very simplistic, purpose-built robots - but they provide consistent, convenient, low-cost service and customers have grown to trust them. Financial services and technology companies are using robotics and AI to address key pressure points, reduce costs and mitigate risks. They are targeting a specific combination of capabilities such as social and emotional intelligence, natural language processing, logical reasoning, identification of patterns and self-supervised learning, physical sensors, mobility, navigation and they are looking far beyond replacing the bank teller. 


\section{- Cloud Computing}

With hybrid cloud, banks have the flexibility and benefits of both private and public cloud, while addressing data security, governance and compliance. The benefits of a hybrid cloud include reduced costs, improved operational efficiency and enhanced innovation. Financial institutions will need to manage a lot of different forms of data, and much of it will be beyond your control, in forms and locations that they have not anticipated. The cloud offers a good use case. For example, financial institutions may turn to hybrid cloud brokers to help them improve the way they aggregate data, analyse activity, and react to user behaviour through a single customer view for customer banking. This integration is essential for front office applications and channel architecture domains that expose such services to their customers. This is another reason that cloud computing is becoming so dominant: it makes it much easier to manage and analyse 'big data'. Internally, it allows financial institutions to design and deliver their financial services more quickly.

- Cost optimization through paper less transactions

Use of digital e-banking and m-banking will open new opportunities for both cost reduction and revenue growth. This will assist in optimizing cost with the use of paper less transactions.

\section{Conclusion}

While there are many concerns, the fact that financial services businesses are investing in technology like AI and machine learning, as well as still planning to grow internationally, means that they are providing themselves with the best chances of dealing with any upcoming challenges effectively. Financial services have to face emerging competitors, shifting demographics, rising customer expectations and changing regulations. Technology offers solutions, allowing financial institutions to cut costs and become more efficient at what they do. But, most technology is not proprietary, so it is a bit of a race: if financial institutions blink, they might find that their competitor has already built up advantages that are now harder for them to match. As we see it, most financial institutions are currently focusing much of their IT attention on the short-term. We understand this: on any given day, there are fires to fight, with regulatory fixes, fraud attempts, budget discussions and so on. Each financial institution will respond to these trends and priorities in their own way, largely dependent on their unique position in the market, desired path forward, brand positioning, regulatory circumstances, and organisation capabilities. Financial service companies are having various opportunities and challenges which they are facing today due to the rapid digital innovations.

\section{References}

Bastid, V., Hawes, A., and Chitra, T. (2016, February). Digital transformation: The challenges and opportunities facing banks, Oracle Efma. Retrieved April 5, 2021.

Burlakov, G. (2021, March 12). 10 Challenges for the Financial Services Industry, Technorely. Retrieved April 5, 2021.

IFC. (2017, August). Digital Financial Services: Challenges and Opportunities for Emerging Market Banks. Retrieved April 4, 2021. 
Linchpin. (2021, March 6). Financial Services Industry Challenges \& Opportunities In 2021, Linchpin. Retrieved April 4, 2021.

Marous, J. (2021). 10 Technologies That Will Disrupt Financial Services In The Next 5 Years, The Financial Brand. Retrieved April 5, 2021.

McCreadie, P. (2021, January 25). Opportunities and challenges facing financial services firms in 2021, Global Banking and Finance Review. Retrieved April 5, 2021.

PWC. (2021). Financial Services Technology 2020 and Beyond: Embracing disruption. Retrieved April 9, 2021.

Success Factors (2021). Workforce 2020: Challenges and Opportunities for Financial Services. Retrieved April 4, 2021.

Vijai, C. (2019). Fintech in India - Opportunities and Challenges. South Asian Academic Research Journals, 8(1), 42-54. Retrieved April 3, 2021. 